\title{
PERFILES MOTIVACIONALES DEL ALUMNADO DE EDUCACION SECUNDARIA Y RENDIMIENTO ACADEMICO
}

\section{Alfonso Barca Lozano}

CDD: 153.4

\section{RESUMEN}

En este artículo se señalan las principales variables y perfiles motivacionales que describen al alumnado de educación secundaria en Galicia (España). Para ello partimos de dos criterios básicos: por una parte, se analiza la percepción de los alumnos sobre las relaciones familiares padres/hijos en cuanto a la valoración que hacen los padres de la capacidad y del trabajo escolar de los hijos y, por otra parte, se busca obtener esa misma percepción del alumnado a partir de la valoración de su propio rendimiento académico alto y bajo. Finalmente, se pretende establecer una línea secuencial, por orden de prioridad, de aquellos indicadores motivacionales que tienen una mayor capacidad descriptiva del alumnado y poseen un efecto significativo sobre el rendimiento y aprendizaje del alumnado de educación secundaria.

\section{PALABBRAS CLAVE}

Estrategias cognitivas; Metas de aprendizaje; Metas de rendimiento; Metas de valoración social; Estilos de aprendizaje; Rendimiento académico

\section{MOTIVATIONAL PROFILES OF SECONDARY EDUCATION STUDENTS AND ACADEMIC ACHIEVEMENT}

\begin{abstract}
In this study, the main motivational variables and profiles which describe Galician (Spain) Secondary School pupils are shown. In order to do so, we start from two basic criteria: on one hand, the way in which these pupils perceive the parent-children relationship with regard to how parents value their children's capacity and their school work is analysed, and on the other hand, starting from the way in which pupils value their own high or low academic achievement, we aim to obtain the same perception as above on the part of the pupils. Finally, the aim is to establish a sequential line, by order of priority, of those motivational indicators which best describe the pupils and which have a significant effect on Secondary School pupil's achievement and learning.
\end{abstract}

\section{KEYWORDS}

Cognitive strategies; Performance objectives; Achievement goals; Social value objectives; Learning styles; Academic achievement 


\section{INTRODUCCION}

Para la elaboración de este capítulo tomamos las conclusiones más relevantes que se han obtenido de diferentes investigaciones realizadas con alumnado de educación secundaria a lo largo de la última década bajo la dirección del autor de este trabajo.

Desde el año 1999 en el que se ha comenzado a trabajar sobre los procesos de aprendizaje que afectaban al rendimiento académico del alumnado de educación Secundaria de Galicia (España) y hasta fechas muy recientes que se ha iniciado un proyecto de investigación conjunta en colaboración con colegas de Portugal (ALMEIDA, 2008) sobre la identificación de indicadores y determinantes de aprendizaje y desarrollo en el alumnado de educación secundaria con muestras procedentes de ciudades del Norte de Portugal (Porto, Braga y Chaves), así como de las ciudades de A Coruña, Santiago de Compostela, Pontevedra y Ourense, en Galicia, hemos podido identificar una serie de determinantes e indicadores psicológicos que forman parte de una secuencia pautada de procesos cognitivos y motivacionales en relación con el rendimiento académico bajo y alto, asociado con la percepción de valoración y satisfacción familiar positiva y negativa de la capacidad y del esfuerzo en el trabajo escolar que el alumnado de educación secundaria percibía en sus relación con el rendimiento y con sus relaciones familiares.

\section{ANALISIS CORRELACIONAL ENTRE VARIABLES FAMILIARES Y RENDIMIENTO ACADEMICO}

$\mathrm{Si}$ se analizan con detenimiento las correlaciones existentes entre las variables relacionales-familiares en relación con el rendimiento académico, entendido en la línea conceptual de González-Pienda (2003), en las cinco últimas investigaciones de las que se toman los datos y se presentan en este capítulo, podemos observar, en primer lugar, que el rendimiento medio académico del alumnado mantiene, en todas las investigaciones estudiadas y realizadas por nosotros, altas correlaciones positivas y significativas con variables familiares como son: satisfacción familiar del rendimiento/trabajo escolar o valoración del estudio por parte de la familia, o valoración positiva de la capacidad y esfuerzo (véase tabla 1 de muestras analizadas). 


\section{TABLA 1}

Muestras de los sujetos participantes por sexos y niveles académicos integrados en las investigaciones realizadas

\begin{tabular}{|c|c|c|c|c|c|}
\hline & \multicolumn{5}{|c|}{ PARTICIPANTES/MUESTRAS } \\
\hline & (1) G/1999 & (2) G/2002 & $\begin{array}{l}\text { (3) G/TB/ } \\
\text { y Morán }\end{array}$ & Almei & (5) Muestra Total \\
\hline $\mathrm{N}$ & 393 & 1.392 & 1.386 & & 3.898 \\
\hline Sexo: hombres & 189 & 719 & 541 & & 1.653 \\
\hline Sexo: mujeres & 204 & 673 & 845 & & 1.722 \\
\hline $1^{\circ}-2^{\circ} \mathrm{ESO}$ & 150 & 726 & & & 876 \\
\hline $3^{\circ}-4^{\circ} \mathrm{ESO}$ & 243 & 666 & 841 & & 1.750 \\
\hline $1^{\circ}-2^{\circ}$ Bachillerato & & & 545 & & 545 \\
\hline $1^{\circ}, 2^{\circ}, 3^{\circ}$ de ESO & & & & 727 & 727 \\
\hline
\end{tabular}

*Datos aportados en la investigación realizada por: (1) A. Barca (1999a) con una muestra representativa de alumnado de los 4 cursos de la ESO (Alumnado de Educación Secundaria) (Proyecto Xupro/Galicia, 1999); (2) Datos de A. Barca y M. Peralbo (2002), Proyecto Feder-2002, correspondiente a una muestra de estudiantes de Educación Secundaria (ESO) de Galicia. (3) Datos de J. C. Brenlla correspondientes a su tesis doctoral (2005) y Morán (2004) con una muestra de estudiantes de Educación Secundaria (ESO) y de Bachillerato de Galicia. (4) Muestra de alumnado de Portugal y Galicia de Educación Secundaria (ESO). (5) Total Muestra de Galicia/España y Portugal.

Todos estos datos implican el hecho de que el rendimiento académico es más alto en la misma medida en que también lo son las percepciones del alumnado sobre la satisfacción de su familia con los resultados positivos que obtiene, el interés motivacional por las propias tareas y por el aprendizaje en sí mismo, por la colaboración estrecha de la familia con el centro escolar y la motivación por terminar con éxito los estudios y obtener un buen trabajo en el futuro (ver tabla 2). 


\section{TABLA 2}

Índices de Correlación entre los Factores de la Escala CDPFA (Subescala Variables Familiares: Valoración/Satisfacción familiar de la capacidad y esfuerzo y Nota Media Global (BARCA, 1999; BARCA Y PERALBO, 2002; MORÁN, 2004; BRENLLA, 2005; ALMEIDA, 2008)

\begin{tabular}{lcccccc}
\hline Factor & $\begin{array}{c}\text { Barca } \\
(1999)\end{array}$ & $\begin{array}{c}\text { Barca y Peralbo } \\
(2002)\end{array}$ & $\begin{array}{c}\text { Morán } \\
(2004)\end{array}$ & $\begin{array}{c}\text { Brenlla } \\
(2005)\end{array}$ & $\begin{array}{c}\text { Almeida } \\
(2008)\end{array}$ \\
\hline $\begin{array}{l}\text { Valoración/Satisfacción familiar de } \\
\text { la capacidad y esfuerzo/trabajo escolar }\end{array}$ & $0,531^{* *}$ & $0,584 * *$ & $0,308^{* *}$ & $0,638^{* *}$ & $0,299^{* *}$ \\
$\begin{array}{l}\text { Refuerzo familiar del rendimiento } \\
\begin{array}{l}\text { Ayuda de la familia al trabajo escolar } \\
\text { y colaboración Familia/centro/ }\end{array}\end{array}$ & 0,043 & $-0,031$ & 0.010 & $0,055^{*}$ & 0.060 \\
\hline
\end{tabular}

Expectativas negativas familia

$* \mathrm{p}<.05 \quad * * \mathrm{p}<.01 \quad$ La correlación es significativa al nivel 0,01 (bilateral).

Sin embargo, hay que destacar, por otra parte, las variables de correlación negativa y significativa con el rendimiento académico. Se observa que en la práctica totalidad de las cinco investigaciones que estamos analizando, tanto las variables: a) refuerzo familiar del rendimiento, b) colaboración familia/centro, como, c) control familiar y ayudas familiares en el estudio (especialmente en el alumnado de FP) guardan correlaciones negativas y significativas o muy bajas correlaciones. Esto significa que el rendimiento académico es más bajo en la misma medida en que son más altos aspectos como la existencia de recompensas/elogios por parte de la familia hacia el sujeto, promesas de regalos o recompensas materiales, haciendo referencia tanto al esfuerzo realizado como a las calificaciones conseguidas. Además, ocurre lo mismo cuando la familia acostumbra a comparar las notas del sujeto con las de sus compañeros/as de clase o la percepción del alumno sobre el control familiar en los estudios o sobre la colaboración familiar con el centro escolar (véase tabla: 1).

En todo caso, la conclusión a la que hemos llegado en este punto ha sido la de que se constata que existe una alta correlación positiva y significativa entre dos variables: el medio/alto rendimiento académico con la percepción que el alumnado posee de la valoración/satisfacción familiar positiva de su capacidad y de su esfuerzo en el trabajo escolar. Del mismo modo se ha constatado el hecho de que existe una muy baja correlación, o bien una correlación negativa y significativa entre el rendimiento académico medio y 
variables como Refuerzo familiar del rendimiento o ayuda de la familia al trabajo escolar o bien colaboración familia/centro escolar. De aquí que, en la secuencia de procesos motivacionales y cognitivos que inciden en el rendimiento escolar hayamos relacionada de una manera estrecha el buen rendimiento académico con variables familiares de satisfacción/valoración de la capacidad y esfuerzo, es decir, del trabajo escolar y, del mismo modo, se han identificado con el bajo rendimiento académico/escolar otras variables familiares como el refuerzo familiar del rendimiento, la colaboración familia/centro o el control familiar y ayudas familiares en el estudio y que presenta o una muy escasa correlación o una correlación negativa con el rendimiento académico-

\section{SECUENCIA DE PROCESOS MOTIVACIONALES, COGNITIVOS Y ESTRATEGIAS DE APRENDIZAJE CON INCIDENCIA EN EL RENDIMIEMTO ACADEMICO}

A partir de las investigaciones realizadas en la última década por el equipo y autores de esta Comunicación pasamos a describir los perfiles dominantes de procesos motivacionales y cognitivos del alumnado de educación secundaria, especialmente partiendo del criterio de alumnado con rendimiento académico bajo y percepción de relaciones familiares deficientes y alumnado con rendimiento académico medio/alto y percepción positiva de relaciones familiares con el medio escolar y personal de dicho alumnado. Para la elaboración de dichas secuencias (ver cuadros 1 y 2) se han utilizado los resultados y conclusiones de los trabajos de investigación de Barca (1999), Barca y Peralbo (2002), Morán (2004), Mascarenhas (2004), Brenlla (2005) y Almeida (2008).

Como caracterización general y avanzando perfiles motivacionales y cognitivos, de estrtategias de aprendizaje de carácter psicosocial y psicoeducativo del alumnado de educación secundaria de niveles de aprendizaje deficientes y rendimiento académico bajo, con relaciones familiares negativas, por una parte, y por otra, pasamos, del mismo modo, a ofrecer los mismos perfiles motivacionales y cognitivos y de estrategias de aprendizaje, pero con alumnado de rendimiento académico alto y relaciones familiares positivas.

De esta forma, podemos incluir y a avanzar los siguientes elementos, determinantes o indicadores, procediendo a partir de diversas áreas características referidas a los principales ámbitos especialmente motivacionales, cognitivos y de estrategias de aprendizaje del alumnado de educación secundaria (véanse cuadros 1 y 2). 
A/ EN RELACIÓN CON EL AREA DE INDICADORES PSICOSOCIALES, FAMILIARES Y RELACIONALES

-- Es un alumnado con una percepción de la valoración por parte de su familia baja o muy baja en relación con su trabajo escolar y las expectativas de futuro sobre su trabajo. Es decir: percibe que su familia más próxima, padres/hermanos no valora suficientemente su trabajo escolar en concreto y, en general a su persona.

-- Las condiciones materiales de estudio y trabajo en su casa son deficientes; es decir: las condiciones en relación con el espacio, luminosidad y comodidad, inexistencia en su casa de un lugar fijo de estudio, la escasa disponibilidad de materiales y recursos para su trabajo escolar y la carencia de una planificación adecuada de su trabajo, son otros tantos elementos que están condicionando negativamente su proceso de aprendizaje asi como su rendimiento académico.

-- Es un alumnado al que se le refuerza poco, pero cuando se hace es de forma extrínseca, es decir, se observan influencias negativas cuando su familia tan sólo le refuerza con promesas o se le elogia y/o se le recompensa por su calificaciones o cuando obtiene alguna buena nota; por lo tanto, se concluye que, en líneas generales, el refuerzo extrínseco (material, externo) mantiene una influencia negativa en relación con el aprendizaje y el rendimiento.

-- Por otra parte, este alumnado de educación secundaria de bajos niveles de rendimiento escolar percibe por parte de su familia una valoración negativa de su capacidad. Considera que por parte de la familia no se le valora suficientemente de cara a su capacidad o su esfuerzo para llevar a cabo con éxito las tareas escolares.

-- El uso de criterios comparativos por parte de su entorno familiar es negativo. En efecto, cuando la familia habitualmente trata de comparar las notas de sus hijos con las notas de los compañeros de clase es un hecho que se asocia a menudo con el rendimiento en el aprendizaje, pero con signo negativo. 
B/ EN RELACION CON VARIABLES EMOCIONALES/AFECTIVAS (AUTOCONCEPTO) Y METAS ACADEMICAS.

-- Se trata de un alumnado con niveles bajos de autoconcepto académico general y académico (AAG). En líneas generales podemos calificar a este indicador como una medida de evaluación indicativa de cómo se percibe el alumno a sí mismo como estudiante. Este indicador incluye una valoración de si mismo en las áreas de matemáticas, áreas verbales y resto de asignaturas o materias. Este tipo de autoconcepto se va construyendo no sólo en función de los logros obtenidos sino como consecuencia de un proceso de comparación social con sus compañeros y de las expectativas de sus profesores y sus padres. Es entonces, cuando esta construcción del autoconcepto es débil, negativa o inconsistente y, asi sus influencias en el rendimiento y aprendizaje son también negativas.

-- Es un alumno con bajos niveles de autoconcepto académico. A diferencia de la anterior variable o determinante, diremos que el autoconcepto académico es la representación que el alumno tiene de sí mismo como aprendiz. En consecuencia, el autoconcepto académico se refiere a la idea que el alumno tiene de sus habilidades para llevar a cabo la tarea de aprender y afrontar el aprendizaje en un contexto instruccional, en concreto, en el contexto escolar. Así, se puede hablar de varios tipos de autoconcepto: el matemático, el físico (capacidad y apariencia) y los niveles de estabilidad emocional. Se sabe que cuando estos tipos de autoconcepto están en sus niveles bajos o deficitarios, este hecho afecta negativamente al aprendizaje y, en consecuencia, al rendimiento académico.

-- Metas académicas: es un indicador que trata de determinar hacia qué metas, objetivos o expectativas se orienta la actividad del alumno y, como consecuencia, qué clase de motivación o esfuerzo es el que guía el proceso de aprendizaje. Este factor hace referencia, en concreto, al esfuerzo/trabajo que realiza el alumno y la satisfacción en los estudios. Niveles deficientes o bajos en la capacidad de motivación o esfuerzo del alumno en su proceso de aprendizaje derivarán en un rendimiento académico deficiente. Dominan las metas de tipo de logro o rendimiento, de evitación de fracaso y de valoración social (orientadas al yo: se busca la obtención de rendimiento a corto plazo, con el mínimo esfuerzo y en función de contingencias externas...) frente a las 
metas de aprendizaje o de dominio en las que priman las necesidades de conocer, saber, dominar las materias y formarse de acuerdo con las prestaciones profesionales del futuro en sociedad.

\section{C/ EN RELACION CON LOS ESTILOS ATRIBUCIONALES CAUSALES MULTIDIMENSIONALES}

-- Hay un primer determinante o factor que es el de Atribución a la Capacidad del Rendimiento académico e indica que se trata de un tipo de atribución en el que el lugar de causalidad es interna, en la dimensión temporal es estable y no controlable. Tiene un efecto positivo sobre la motivación de rendimiento, las expectativas de éxito y la autoestima y, en consecuencia, es un indicador/predictor importante del rendimiento académico. En caso contrario, la incidencia en el rendimiento es negativa. Podemos decir que en el alumnado de rendimiento bajo este tipo de atribución apenas aparece como relevante. Es un estilo atribucional recesivo y, sin embargo, aparece como relevante el estilo de atribución al Profesor (PF-BRA), a la baja capacidad (BC-BRA) y a la suerte (S-R).

-- De igual modo que el estilo atribucional causal anterior, el de Atribución al Esfuerzo del rendimiento académico (AE-RA) tiene un efecto positivo sobre la motivación de rendimiento, las expectativas de éxito y la autoestima, así como efectos y determinantes importantes sobre el rendimiento y aprendizaje escolares. El alumno de educación secundaria con bajos niveles de rendimiento parece que no dispone de este tipo de atribuciones (a la capacidad y al esfuerzo), es decir, apenas existen en su repertorio atribucional causal. Es otro tipo de estilo atribucional recesivo en alumnado de rendimiento bajo.

-- Sin embargo, es frecuente y dominante el tipo de atribuciones al azar/suerte (S-R), a la facilidad/dificultad de las materias del rendimiento académico (FM/RA) y la atribución al profesorado (PF-BRA) (no me entiende, es tacaño con las notas, no explica bien..., etc.) entre el alumnado de educación secundaria de rendimiento bajo y percepción deficiente de valoración familiar. Ello significa que, en general, para aquellos alumnos con rendimiento académico bajo y valoración familiar deficiente, la facilidad de las materias, el profesorado y la mala suerte son los indicadores responsables del rendimiento académico de los alumnos y de que su familia no les 
valore su esfuerzo y capacidad. Insistimos que lo mismo ocurre cuando en la atribución se hace responsable del bajo rendimiento al profesorado o a su baja capacidad. Son tipos de atribuciones externas e interna en cuanto al lugar de la causalidad, estables en la dimensión estable-inestable y no-controlables en la dimensión de control/no-control. Tienen efectos negativos para la motivación de logro y la formación positiva del autoconcepto y autoestima de los alumnos.

D/ EN RELACION CON LOS ESTILOS Y ENFOQUES DE APRENDIZAJE (MOTIVOS Y ESTRATEGIAS DE APRENDIZAJE) DEL ALUMNADO DE EDUCACION SECUNDARIA

-- Los alumnos de educación secundaria con bajo rendimiento escolar, en su proceso de adopción y abordaje de sus tareas de estudio y aprendizaje, asocian a menudo los motivos y estrategias de aprendizaje a los Enfoques de Orientación Superficial (EORSP). Utilizan las estrategias de memorización, retención de hechos y procedimientos, sin buscar relaciones internas entre los diferentes contenidos. Esta adopción de enfoques superficiales conducen a la obtención de resultados académicos (rendimiento) negativos o bajos.

-- Al contrario, en ningún caso, estos mismos alumnos adoptan de una manera dominante Enfoques de Orientación al Significado (EOR-SG). Por lo tanto, este tipo de enfoques que se asocian siempre con el rendimiento académico medio-alto o alto. Son alumnos que adoptan enfoques de aprendizaje de tipo comprensivo y significativo, dedicando tiempo al trabajo personal, utilizando estrategias de comprensión, relacionando, optando a buenas calificaciones, de lectura comprensiva, organizando los recursos técnicos -ampliando lecturas, buenos apuntes, organizados y llevados al día--, utilizando debidamente el espacio y el tiempo, en definitiva, implicándose directamente en el proceso de aprendizaje, etc. y que obtienen buenos resultados académicos. Sin embargo, como decimos, en el caso de alumnos con deficientes rendimientos escolares y problemas en la valoración de su persona, su capacidad y esfuerzo por parte se los familiares, en ningún caso este tipo de enfoques llega a ser dominante. 
E/ CAPACIDADES COGNITIVAS/INTELIGENCIA GENERAL Y COMPRENSION LECTORA

-- Una buena dotación en Inteligencia general proporciona en los sujetos habilidades para la resolución de problemas de diverso tipo, capacidades cognitivas básicas, flexibilidad intelectual para trabajar con contenidos mentales diversos, capacidad para la inducción y deducción lógicas, asi como rapidez y agilidad mental para captar relaciones y descubrir leyes y principios. En definitiva, es la inteligencia general un predictor y determinante importante del rendimiento académico. Los alumnos de rendimiento bajo este tipo de capacidades se encuentran en unos niveles medios o bajos. Por lo tanto existe una asociación entre ambos constructos.

-- Resulta determinante al mismo tiempo el nivel de comprensión lectora relacionada con el rendimiento académico. El nivel de comprensión lectora se relaciona significativa y positivamente con todas las materias que cursan los alumnos y con la media global de calificaciones. Un alumno o alumna con buen rendimiento escolar es, en cualquier caso, aquél que tiene un buen nivel de comprensión lectora, de igual modo que alguien con mal rendimiento escolar tendrá, con gran probabilidad, un peor nivel de comprensión lectora.

En resumen, señalamos en los cuadros 1 y 2 la síntesis de lo que se ha afirmado hasta este momento en relación con los perfiles motivacionales, cognitivos y de estrategias de aprendizaje del alumnado de educación secundaria en relación con el rendimiento bajo y medio/alto y con relaciones familiares negativas y positivas. Resultados y conclusiones semejantes se apuntan en trabajos como los realizados por González-Pienda, J.A., Núñez, Alvarez, González-Pumariega, Roces, González, P., Muñiz y Bernardo (2002); Miras, 1996; Harter (1988); Marchesi (2004); Pintrich y Schunk (2006) y Barca (2009).

Explicando brevemente el cuadro 1 hay que decir que aquellos alumnos con rendimiento académico bajo y con una percepción de sus relaciones familiares y de su valoración personal y como alumno deficientes, suelen poseer unas pautas y determinantes motivacionales en los que sobresalen los Enfoques/estilos de aprendizaje de orientación superficial (EOR-SP), con sus motivos y estrategias superficiales. 


\section{CUADRO 1}

Secuencia pautada y perfil de procesos motivacionales, estrategias de aprendizaje y habilidades cognitivas y lectoras en función del bajo rendimiento académico y la percepción negativa y deficiente de valoración familiar del esfuerzo y la capacidad

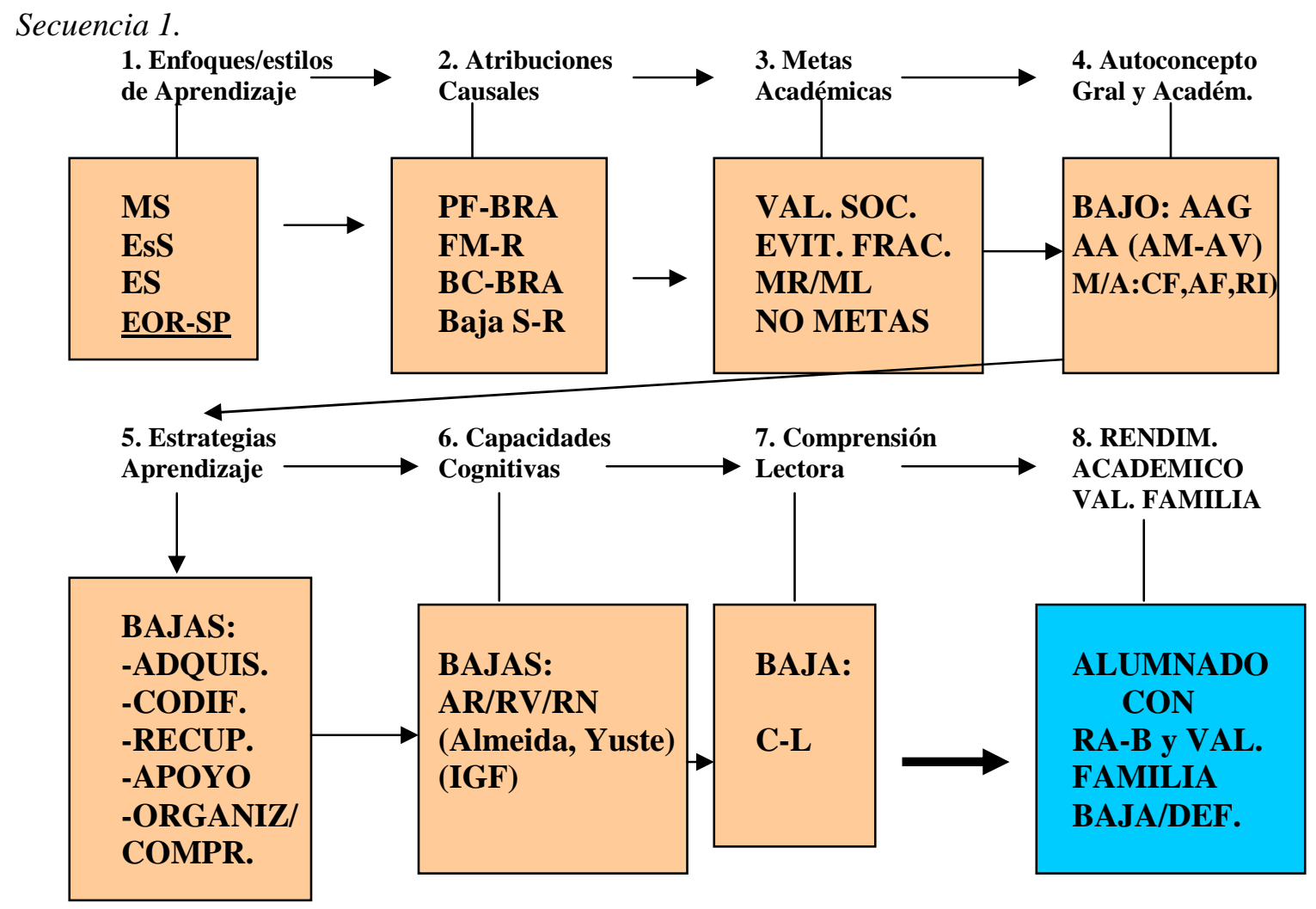

EXPLICACION SIGLAS: MS: Motivo superficial; Es: Estrategia superficial; ES: Enfoque superficial; EOR-SP: Enfoque de orientación superficial. PF-BRA: Atribución del bajo rendimiento al profesorado; FM-R: atribución del rendimiento a la facilidad de las materias; BC-BRA: Atribuición del rendimiento a la baja capacidad; VAL.SOC.: Metas de valoración social; EVIT. FRAC: Metas de evitación de fracaso; MR/ML: Metas de rendimiento/logro; AAG, AA (AM-AV): Bajos niveles de autoconcepto general, autoconcepto académico (matemáticas y verbal); M/A: CF, AF, RI: Autoconcepto Media/alto de capacidad física, apariencia física y relación con iguales. ADQUIS.: Estrategias de adquisición; CODIF.: Estrategias de codificación; RECUP..: Estrategias de recuperación; APOYO: Estrategias de apoyo; ORGANIZ./COMPR.: Estrategias de organización/comprensión. AR/RV/RN: Bajas habilidades cognitivas de razonamiento abstracto, razonamiento verbal y numérico; IGF: Pruebas de inteligencia general factorial; C-L: Bajos niveles de comprensión lectora. RA-B: Alumnado con rendimiento académico bajo y valoración familiar pobre/baja o deficiente.

Al mismo tiempo se sigue un perfil-tipo de atribuciones causales dominantes de atribución de su bajo rendimiento académico al profesorado (no me entiende, no me explica bien, da malas notas....), con unas metas académicas en las que sobresalen las de valoración social, orientadas al "yo", de evitación de fracaso o, en todo caso, de rendimiento/logro y, a veces, no existen metas. 
Los niveles de autoconcepto (autoconcepto académico y general) de este alumnado también son bajos, con una valoración pobre de sí mismos como personas y como alumnos/as, aunque con buenos niveles de capacidad física, percepción positiva de su apariencia física y relación con sus iguales. Además utilizan mal o con deficiencia las estrategias cognitivas de aprendizaje y, en concreto, las estrategias cognitivas de organización y comprensión.

\section{CUADRO 2}

Secuencia pautada y perfil de procesos motivacionales, estrategias de aprendizaje y habilidades cognitivas y lectoras en función del medio/alto rendimiento académico y la percepción positiva y Secuencia 2. óptima de valoración familiar del esfuerzo y la capacidad

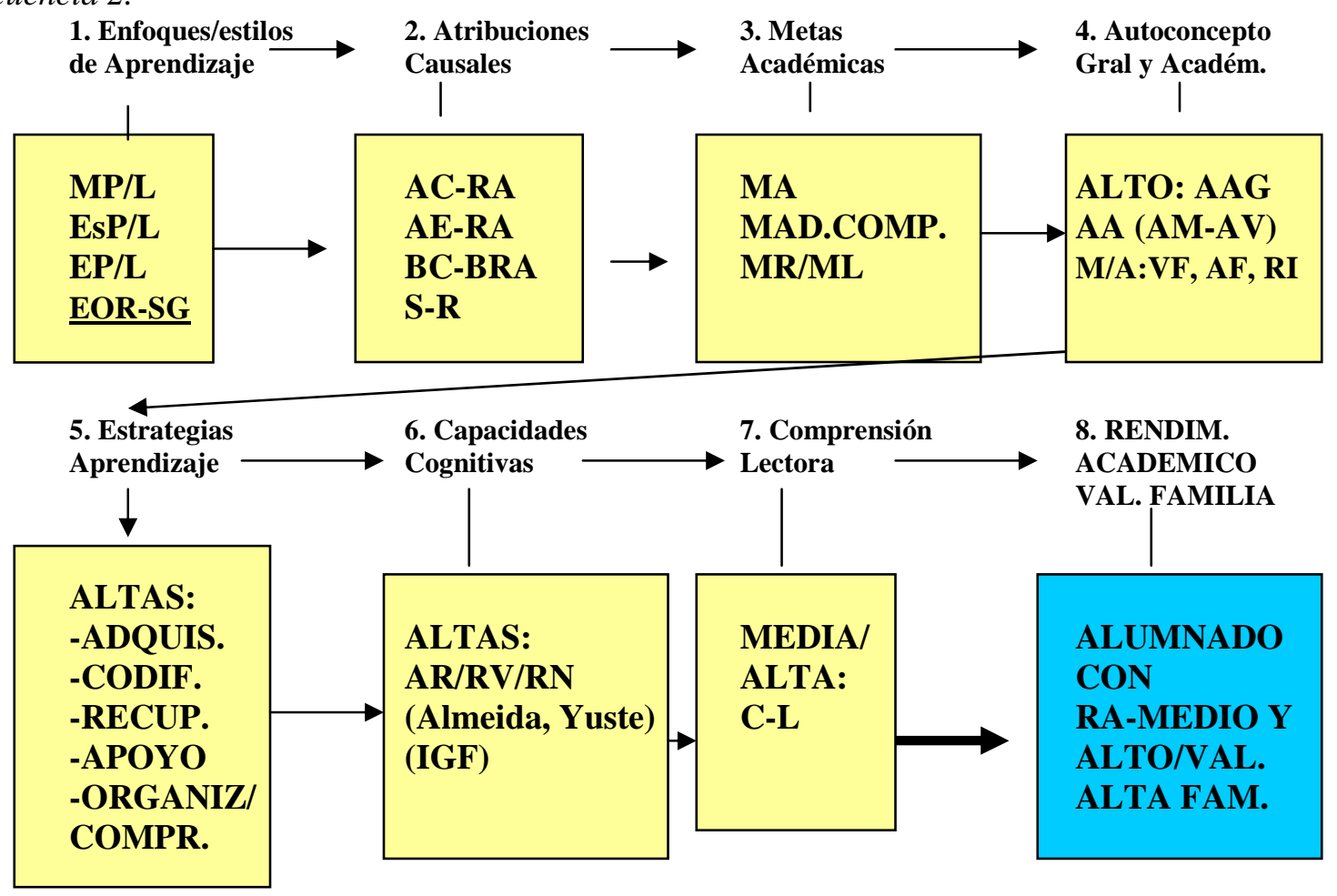

EXPLICACION SIGLAS: MP/L: Motivo profundo y de logro; EsP/L: Estrategia profunda y de logro; EP/L: Enfoque profundo y logro; EOR-SG: Enfoque de orientación al significado. AC-ARA: Atribución del rendimiento a la capacidad; AE-ARA: Atribución del rendimiento al esfuerzo; S-R: Atribuición del rendimiento a la suerte; MA: Metas de aprendizaje; MAD.COMP.: Metas de adquisición de competencias/comprensión; MR/ML: Metas de rendimiento y metas de logro; evitación de fracaso; MR/ML: Metas de rendimiento/logro; AAG, AA (AM-AV): Altos niveles de autoconcepto general, autoconcepto académico (matemáticas y verbal); M/A: CF, AF, RI: Autoconcepto Media/alto de capacidad física, apariencia física y relación con iguales. ADQUIS.: Altos niveles de Estrategias de adquisición; CODIF.: Estrategias de codificación; RECUP.: Estrategias de recuperación; APOYO: Estrategias de apoyo; ORGANIZ./COMPR.: Estrategias de organización/comprensión. AR/RV/RN: Altos niveles de habilidades cognitivas de razonamiento abstracto, razonamiento verbal y numérico; IGF: Pruebas de inteligencia general factorial; C-L: Altos niveles de comprensión lectora; RA-A: Alumnado con rendimiento académico alto y buena/alta valoración familiar. 
Por otra parte, suelen obtener niveles medios y bajos de capacidades cognitivas de razonamiento verbal, numérico y abstracto, mientras que los niveles de comprensión lectora (C-L) son bajos o muy bajos... y, como consecuencia, el rendimiento académico/escolar es deficiente asociado con niveles negativos de valoración familiar respecto de sus capacidades y su trabajo escolar (ver cuadro 1).

Por el contrario, y explicando el cuadro 2 que se inserta en esta página, hay que decir que aquellos alumnos con rendimiento académico medio/alto y relaciones familiares buenas relacionadas con la valoración de su esfuerzo y capacidad y de su trabajo escolar, suelen poseer unas pautas motivacionales en las que sobresalen los Enfoques/estilos de aprendizaje de orientación profunda, hacia la comprensión/significado (EOR-SG), con sus motivos y estrategias profundas y de logro, siguiendo al mismo tiempo, un tipo de atribuciones causales dominantes relacionadas con su buen rendimiento académico y atribuídas a su capacidad y a su esfuerzo, y en parte a la suerte; desarrollando unas metas académicas en las que sobresalen las de aprendizaje, de adquisición de competencias, y, también las de rendimiento/logro (véase cuadro 2).

Los niveles de autoconcepto de este alumnado son medios/altos, con una valoración óptima de sí mismos como personas y como alumnos, aunque con buenos niveles de capacidad física, apariencia física y relación con sus iguales. Además utilizan con frecuencia y de forma adecuada las estrategias cognitivas de aprendizaje y, en concreto, utilizan bien las estrategias cognitivas de organización y comprensión. Por otra parte, suelen obtener niveles medios y altos de capacidades cognitivas de razonamiento verbal, numérico y abstracto, mientras que los niveles de comprensión lectora son medios y altos... y, como consecuencia, el rendimiento académico/escolar es medio y alto, asociado con niveles positivos de percepción de valoración familiar respecto de sus capacidades y su trabajo escolar (ver cuadro 2).

Estos datos que acabamos de aportar coinciden con otras investigaciones realizadas en contextos educativos y culturales diferentes. Por ejemplo González y Tourón, a partir de los trabajos de Harter (1988) señalan cómo hay alumnos que se encuentran atrapados en un círculo negativo que obstaculiza el progreso en el aprendizaje y el rendimiento escolar (GONZALEZ; TOURON, 1994, p. 364-365). Continúan estos autores señalando que si su 
rendimiento académico es bajo se perciben como relativamente incompetentes y se sienten mal acerca de su desempeño escolar, lo cual produce una orientación motivacional más extrínseca y, así, evitan el reto a favor del trabajo más fácil y cómodo, muestran poco interés o placer hacia el aprendizaje y tienden a realizar lo mínimo que el sistema escolar exige. Todo este proceso es el activo promotor que conduce a niveles más bajos de rendimiento escolar (GONZALEZ; TOURON, 1994, p.365).

Por el contrario, los alumnos con buen rendimiento escolar tienden a percibirse altamente competentes y, en consecuencia, experimentan reacciones positivas hacia su trabajo escolar y se sienten bien en relación con su actuación académica. Estas reacciones emocionales y afectivas proporcionan muevos estímulos para el aprendizaje escolar. Se sienten a gusto en su trabajo académico y es posible que estén motivados intrínsecamente y que muestren curiosidad por aprender. Su orientación motivacional conduce a estos alumnos a un nivel más alto de rendimiento.

Para los lectores que deseen ampliar la información sobre los mecanismos psicológicos y psicosociales que están implicados en ambos procesos motivacionales que inciden en el rendimiento académico alto y bajo del alumnado de educación secundaria descritos hasta aquí, recomendamos la lectura de un excelente trabajo realizado por Peralbo, Sánchez y Simón (1986) sobre motivación y aprendizaje escolar desde la teoría de la autoeficacia de A. Bandura.

\section{CONSIDERACIONES FINALES, SUGERENCIAS Y LINEAS DE INTERVENCION PSICOEDUCATIVA}

Conocemos los Informes sobre Educación de la OCDE-2006/2008, llamados Informes PISA (Programa Internacional de Evaluación de Estudiantes) dirigidos por el profesor Andreas Schleicher, Informes que a través de unas 275.000 pruebas directas a otros tantos estudiantes de educación secundaria, compara los resultados educativos de los países, unos 40 aproximados, de la OCDE. Según se revela en estos Informes existen tres grandes áreas o materias importantes en las que nuestros alumnos obtienen un rendimiento pobre y preocupante en relación con la mayoría de los países de la OCDE: comprensión lectora, matemáticas y área de ciencias (química, biología, física). Además se señala que el $23 \%$ y el $25 \%$ aproximado de este alumnado no consigue los conocimientos mínimos necesarios en 
estas materias. Bien: son datos, como se podrá observar, y como ya hemos indicado anteriormente, son coincidentes con otros que hemos obtenido en diferentes trabajos de investigación realizados en al última década y, en concreto, en los años 1999, 2002, 2004, 2005, 2008 y 2009 (BARCA, 1999; BARCA; PERALBO, 2002; MORAN, 2004; BRENLLA, 2005; ALMEIDA, 2008; BARCA, 2009).

En esta línea, las principales aportaciones que nos proporcionan estos estudios promovidos por los Informes PISA de la OCDE y que cada dos años nos emiten sus principales resultados y conclusiones podemos resumirlas analizando las principales líneas educativas de los países que mejores resultados obtienen.

Los expertos que elaboran los Informes afirman que los países que mejores resultados obtienen como Finlandia, Noruega y Suecia, son aquellos que:

- consideran al niño/a y a los jóvenes como el centro del proceso educativo y que vuelcan todas las estrategias formativas para conseguir su éxito su mejor integración en la sociedad;

- son aquellos que le dan un gran valor e importancia a la educación;

- que animan y en algunos casos incentivan, la implicación de las familias en la educación escolar de sus hijos;

- y que dedican un notable volumen de recursos públicos;

- que le dan una gran importancia al papel de los docentes (profesorado);

- que confían en la autonomía de los centros...

Uno de los más relevantes por destacar en todas las materias evaluadas, lo tenemos en Finlandia. En este país, el sistema educativo:

- goza de una alta consideración social que se expresa en medidas tales como que su gasto educativo es el 6,24\% de su PIB,

- la escuela pública es mayoritaria, 
- la institución escolar se entiende como una comunidad de aprendizaje,

- existe una gran descentralización administrativa que se complementa con una importante autonomía de los centros,

- la ratio profesor/alumno es baja

- existe un tratamiento de la diversidad por interese,

- se tiene un gran respeto y estima hacia el profesorado que se observa por su fuerte formación que éstos reciben y en el hecho de que a los más competentes se les sitúa en los primeros cursos de primaria,

- los centros están dotados de enfermerías pediátricas, de psicólogos y trabajadores sociales y se da una orientación escolar efectiva; tienen una importante red de bibliotecas escolares atendidas por personal especializado...

Bastaría con analizar estos datos para comparar nuestra situación con la de estos países de la OCDE para deducir cuáles son nuestras carencias y actuar en consecuencia.

Como propuesta de intervención psicoeducativa de carácter general hay que decir que es preciso contar con una serie de medidas que deben tenerse en cuenta y ejecutarse de modo coordinado y sistémico por parte de la administración educativa (cuerpo de inspectores educativos), los departamentos de orientación de los centros, profesorado/tutores, alumnado afectado, siempre en relación con su familia y grupo/amigos/compañeros de iguales y, en general los medios de comunicación multimedia, especialmente la televisión publica y las televisiones privadas en beneficio del cuidado y atención de nuestros alumnos escolares de los primeros años y de nuestros adolescentes en edades críticas de desarrollo y educación.

Las normas e incidencias que a continuación se exponen, van dirigidas a cada uno de estos sectores, pero deberán entenderse que las medidas que se proponen afectan por igual a todos y cada uno de los elementos que forman e integran el contexto educativo de nuestros alumnos, sobre todo de aquellos con pobres rendimientos escolares. 
En concreto como medidas relevantes de atención a la diversidad habrá que tener en cuenta varios aspectos, entre ellos, a las Instituciones educativas y Profesorado, por una parte y, por otra al Alumnado implicado en el rendimiento académico bajo, a la familia y al grupo de iguales.

\section{Instituciones educativas y profesorado del alumnado de educación secundaria}

a/ Establecer currículos más flexibles y abiertos de modo que se tengan en cuenta los intereses y capacidades de los alumnos.

b/ Ello permitirá contar con una enseñanza más ceñida y ligada a la realidad y a la vida diaria del propio alumno, de manera que sus esfuerzos (sean pocos o muchos) los considere como algo positivo, próximo a sí mismo y les dé un sentido.

c/ Los departamentos de orientación de los centros deben de cumplir unos objetivos y realizar unas actividades siempre en coordinación con el profesorado y tutores de los propios centros, por lo tanto se pide una mayor actividad y refuerzo de su papel hacia el profesorado, tutores y alumnos afectados potenciando la formación permanente del profesorado, incentivando su interés por una enseñanza más individualizada, proponiendo nuevos recursos y medios técnicos, especialmente el acceso a la red internet y las nuevas técnicas de información y la comunicación.

d/ Tener en cuenta que el trabajo, dedicación, cooperación, esfuerzo y conocimientos adquiridos por el alumno son otros tantos elementos convertidos en valores que realmente cuentan a la hora de valorar y evaluar las tareas escolares, en consecuencia hagamos que los propios alumnos adquieran experiencia de éxito sobre estos mismas tareas escolares que se les proponen como su trabajo diario escolar.

e/ Es preciso por otra parte contar con profesionales que ayuden al conjunto de profesores a guiar, dar pautas de orientación, asesorar y ayudarles en caso de situaciones conflictivas, de convivencia, de adaptación o de conductas no deseadas para el grupo escolar de manera que se puede contar con psicopedagogos, educadores sociales, psicólogos que faciliten la labor y asesoren en situaciones complejas o conflictivas. 
f/ En aquellos centros en los que los alumnos que provienen de medios o realidades de inmigración u otros contextos de marginación, es necesario favorecer en la medida de lo posible programas intensivos de educación intercultural facilitando siempre la adaptabilidad, inserción en el grupo y la integración en la comunidad educativa del centro escolar.

g/ Es necesario un mayor control del trabajo del alumno en clase, un mayor control de las normas a tener en cuenta en el trabajo cooperativo dando libertades y exigiendo un mayor caudal de responsabilidades al alumnado y a las familias. Es decir, hay que evaluar de un modo formativo, contínuo, analizando donde están los fallos al no conseguir los objetivos previstos y darlos a conocer tanto a las familias como a las instancias superiores (inspecciones u otros profesionales de la educación...) solicitando ayuda en la responsabilidad educativa de los alumnos implicados.

h/ Fomento de la lectura en nuestros adolescentes/jóvenes: a través de los diferentes medios de comunicación se deberá fomentar de una manera decisiva el gusto, atracción, y adicción por la lectura porque sabemos que de su práctica, actividad y ejercicio provienen una serie importante de beneficios que reinciden en un incremento de conocimientos y mejoras cognitivas. Es preciso concienciar a la administración educativa general y a las Comunidades autónomas particularmente de que esta medida es imprescindible para incrementar muy sensiblemente a los lectores jóvenes. Los jóvenes leen poco y cada vez leen menos. No hablamos de libros de literatura o revistas más o menos científicas que seria la lectura ideal, sino que nos referimos ya a la prensa diaria, a los diarios deportivos, a los comics, a los massmedia (revistas, semanarios, también comics). No se lee en los parámetros que cabría esperar en España y en Galicia entre la gente joven. Son datos que ahora conocemos y que periódicamente se vienen aportando a través de los diferentes observatorios sociológicos que nos llevan a una constante preocupación entre los medios educativos y culturales.

i/ Por el contrario, nos encontramos en la predominancia de la cultura de la imagen en movimiento a través de la televisión, video, DVD o cine en diversos formatos. Programas de televisión con un incremento constante de imágenes de violencia y que, se sabe, son nocivas para la salud psicológica y equilibrio personales de los adolescentes (12-16 años). Pensemos que hay programas de televisión frente a los cuales los alumnos de educación primaria y secundaria pasan varias horas al día sin otros objetivos que tragar todo aquello que ven a 
través de la pequeña pantalla sin unos parámetros críticos y selectivos. Decimos tragar porque son programas no aptos para estas edades y mucho menos para pasar varias horas al día, solos, con ellos. Y aquí llamamos a la colaboración y responsabilidad de las familias. Hay que implicarse en este tema de una manera decisiva e incontestable: no se puede perder más el tiempo en este debate. Es urgente y necesario el remedio.

j/ De una manera imperiosa porque estamos en el siglo XXI, en Europa, es preciso, de una vez por todas, incrementar sensiblemente la inversión económica en educación: se debe dignificar el trabajo desarrollado por todos los profesionales de la educación de todos los niveles educativos. Insistimos mucho sobre este hecho, pero es que resulta absolutamente incontestable: proveer de mayores, mejores y significativas retribuciones al profesorado, dotarle de mayores y mejores recursos humanos, con una mayor dotación de profesionales de la Psicopedagogía y Psicología, además de los instrumentos que faciliten y no entorpezcan la labor del profesorado (acceso a los multimedia, a las redes de internet, mayores dotaciones de ordenadores, etc.). El eje en torno al cual gira siempre la mejora de la calidad en educación pasa por la dignificación de la labor del profesorado. Así ha ocurrido en todos los países, asi es y asi será en el futuro.

k/ Se trata, en definitiva, de un incremento significativo de la inversión en educación, no se trata de un gasto en educación, se trata de una inversión. Mientras olvidemos este principio, en nuestro país se darán los desajustes educativos observados en la actualidad... y, pensemos que se trata de nuestro inmediato futuro como ciudadanos libres y avanzados en una sociedad que camina cada vez más hacia altas cotas humanísticas, técnicas y científicas. Preparémonos para hoy y para mañana. No se puede exigir más al profesorado. Sin duda está haciendo y desarrollando con absoluta responsabilidad y profesionalidad, con los mimbres de que dispone, sus funciones educativas e instruccionales. 
Para el alumnado implicado en el rendimiento académico bajo, para la familia y grupo de iguales

Desde la perspectiva más personalizada, familiar y de grupo de iguales y centrándonos en el alumno con fracaso escolar, con dificultades de aprendizaje, de adaptación o integración en el grupo, con problemas de conducta y/o con niveles pobres de rendimiento escolar habrá que establecer pautas por parte del propio centro escolar, vía departamento de orientación y vía de tutores/familia, que vayan dirigidas a:

a) Fomento y mejora del autoconcepto y autoestima positivas, facilitando lo máximo posibles técnicas y estrategias aprendizaje y de estudio (subrayados, síntesis, resúmenes....fomento diario e insistente de la lectura, técnicas de instrucción directa, estudios y trabajos dirigidos), pautas de asertividad y habilidades sociales...

b) Estableciendo la metodología y la planificación y disciplina necesaria en la realización del trabajo escolar y tareas de estudio diario por parte del alumno en casa.

c) Control de las horas de visionado de TV en tiempos no adecuados para la visión de programas que no están dirigidos estrictamente a sus intereses. No se puede permitir la cultura televisiva como una pérdida de tiempo; es preciso buscar alternativas deportivas, de juegos al aire libre, de juegos de grupo... Nunca se abandonará a los hijos a un descontrol de visionado televisivo, sin más. Es nuestra responsabilidad importante como padres.

d) Se potenciarán las actividades de atención/concentración, las de motivación, especialmente haciendo ver la importancia de establecer metas a corto y a medio plazo. 
e) Estimular siempre el interés, superación y solidaridad ante las dificultades, asi como su interés por la estabilidad emocional y afectiva. Es necesario facilitar el diálogo y el interés por la comunicación entre los miembros de la familia; con ello se facilita el interés por la interacción con el grupo y el gusto por la conversación, la narración, el contar historias, favorecer y ayudar a otros a aprender: ese es el verdadero sentido de la educación tanto en la familia como en los niveles de educación formal o escolar.

f) Potenciar el trabajo en equipo, el trabajo en grupo, la cooperación, la solidaridad. Lo importante es considerar que se está avanzando hacia un crecimiento personal desde la óptica de la triple dimensión conductual de la persona: la cognitiva/competencial, afectiva/emocional y social/interaccional. Todo lo que sea mejorar estas tres áreas y cuidar de su crecimiento personal tendrá siempre, como resultado, un efecto educativo.

\section{REFERENCIAS}

ALMEIDA, L. Percurso escolar na adolescência: sucesso/insucesso e

Continuidade/abandono. Proxecto de investigación. Centro de Estudos Euro-Regionais (CEER), Universidade do Minho, Braga, 2008/2009.

BARCA, A. Análisis causal y transcultural de los enfoques y estrategias de aprendizaje en el alumnado de Educación Secundaria en Galicia y Puerto Rico. Santiago de Compostela: [s. n.], 1999. (Proyecto de investigación. Comisión Asesora de Investigación Científica y Técnica de la Consellería de Educación-Xunta de Galicia (C.A.I.C.Y.T.-G). Código: Proxecto XUGA 10601B97). (D.O.G. 12/08/97).

; Motivación y aprendizaje en contextos educativos. Granada: Grupo Editorial Universitario, 2009.

BARCA, A.; PERALBO, M.; J. C. BRENLLA. Atribuciones causales y enfoques de aprendizaje: la Escala SIACEPA. Psicothema, Oviedo, v. 16, n. 1, p. 94-103, 2004. 
BARCA, A.; PERALBO, M. Proyecto FEDER (1FD97-0283): los contextos de aprendizaje y desarrollo en Educación Secundaria en Galicia. Madrid: Dirección General de Enseñanza Superior; Ministerio de Ciencia y Tecnología, 1998-2002. (3 vols.).

BRENLLA, J. C. Atribuciones causales, enfoques de aprendizaje, rendimiento académico y competencias bilingües en alumnos de educación secundaria: un análisis multivariable. 2005. Tese (Doctorado em Psicologia) - Universidad de A Coruña, A Coruña, 2005.

GONZALEZ, M. C.; TOURÓN, J. Autoconcepto y rendimiento escolar. Pamplona: EUNSA, 1994.

GONZALEZ-PIENDA, J. A. El rendimiento escolar. Un análisis de la variables que lo condicionan. Revista Galego-Portuguesa de Psicoloxía e Educación, A Coruña, v. 9, n. 7. p. 247-258. 2003.

. et al. Inducción parental a la autorregulación, autoconcepto y rendimiento académico. Psicothema, Oviedo, v. 14, n. 4, p. 853-860, 2002.

HARTER, S. Developmental processes in the construction of the Self. In: YAWKEY, T. D; JOHNSON, J. E. (Ed.). Integrative processes and socialization: early to middle childhood. Hillsdale, New Cork: Lawrence Erlbaum, 1988.

MARCHESI, A. Qué será de nosotros, los malos alumnos. Madrid: Alianza Ensayo, 2004.

MASCARENHAS, S. Avaliação dos processos, estilos e abordagens de aprendizagem dos alunos do ensino médio do Estado de Rondônia (Brasil). 2004. Tese (Doutorado) Universidade da Coruña, A Coruña, 2004.

MIRAS, M. Aspectos afectivos y relacionales en los procesos de interacción educativa. In: BARCA, A.; GONZÁLEZ-PIENDA, J. A.; GONZÁLEZ, R. J. Escoriza: Psicología de la instrucción. Barcelona: Ediciones Universitarias de Barcelona, 1996.

MORAN, H. Autoconcepto, enfoques de aprendizaje y rendimiento académico en alumnos de formación profesional de Galicia. 2004. Tese (Doutorado em Psicologia) - Universidad de A Coruña, A Coruña, 2004.

PERALBO, M.; SANCHEZ, J. M.; SIMON, M. A. Motivación y aprendizaje escolar.: una aproximación desde la teoría de la autoeficacia. Infancia y Aprendizaje, Madrid, n. 35-36, p. 37-45, 1986.

PINTRICH, P.R.; SCHUNK, D. H. Motivación en contextos educativos: teoría, investigación y aplicaciones. Madrid: Pearson Prentice Hall, 2006. 
Profesor-Catedrático de Psicología de la Educación en la Facultad de Ciencias de la Educación de la Universidad de A Coruña. Publica varios libros y artículos relacionados con los procesos de aprendizaje en relación con el rendimiento académico y sobre dificultades de aprendizaje y procesos motivacionales en contextos educativos. Director de la Revista Galego-

Portuguesa de Psicoloxía e Educación. Ha sido director del Departamento de Psicología Evolutiva y de la Educación (1991-1996), Decano de la Facultad de Ciencias de la Educación (19961998), Secretario General de la Universidad de A Coruña (1998-2001), Vicerrector de la Universidad de A Coruña (2001-2004). Correspondencia: Universidade da Coruña. Facultad de Ciencias de la Educación. Campus de Elviña, s/n. 15071 A Coruña. E-mail: barca@udc.es

Recebido em: 20/07/2009 Publicado em: 30/10/2009 